\title{
Dorsal Horn of Mouse Lumbar Spinal Cord Imaged with CLARITY
}

\author{
Gulgun Sengul, ${ }^{1}$ Huazheng Liang $\mathbb{D}^{2,3}$ Teri M. Furlong, ${ }^{2,4}$ and George Paxinos ${ }^{2,4}$ \\ ${ }^{1}$ Department of Anatomy, School of Medicine, Ege University, Bornova, Izmir 35100, Turkey \\ ${ }^{2}$ Neuroscience Research Australia, 139 Barker St, Randwick, Sydney NSW 2031, Australia \\ ${ }^{3}$ Department of Neurology, Translational Research Institute of Brain and Brain-Like Intelligence, Shanghai Fourth People's Hospital \\ Affiliated to Tongji University School of Medicine, Shanghai 200081, China \\ ${ }^{4}$ School of Medical Sciences, The University of New South Wales, Kensington, NSW 2052, Australia
}

Correspondence should be addressed to Huazheng Liang; huazheng_liang@tongji.edu.cn

Received 17 June 2020; Accepted 30 July 2020; Published 14 August 2020

Academic Editor: Enzo Terreno

Copyright (C) 2020 Gulgun Sengul et al. This is an open access article distributed under the Creative Commons Attribution License, which permits unrestricted use, distribution, and reproduction in any medium, provided the original work is properly cited.

\begin{abstract}
The organization of the mouse spinal dorsal horn has been delineated in 2D for the six Rexed laminae in our publication Atlas of the Spinal Cord: Mouse, Rat, Rhesus, Marmoset, and Human. In the present study, the tissue clearing technique CLARITY was used to observe the cyto- and chemoarchitecture of the mouse spinal cord in $3 \mathrm{D}$, using a variety of immunohistochemical markers. We confirm prior observations regarding the location of glycine and serotonin immunoreactivities. Novel observations include the demonstration of numerous calcitonin gene-related peptide (CGRP) perikarya, as well as CGRP fibers and terminals in all laminae of the dorsal horn. We also observed sparse choline acetyltransferase (ChAT) immunoreactivity in small perikarya and fibers and terminals in all dorsal horn laminae, while gamma aminobutyric acid (GABA) and glutamate decarboxylase-67 (GAD67) immunoreactivities were found only in small perikarya and fibers. Finally, numerous serotonergic fibers were observed in all laminae of the dorsal horn. In conclusion, CLARITY confirmed the 2D immunohistochemical properties of the spinal cord. Furthermore, we observed novel anatomical characteristics of the spinal cord and demonstrated that CLARITY can be used on spinal cord tissue to examine many proteins of interest.
\end{abstract}

\section{Introduction}

The major characteristics of the neurons of each of the spinal dorsal horn laminae have been studied using standard histological, histochemical, and immunohistochemical staining methods. Our mouse spinal cord atlases published in 2009 and 2013 have provided a detailed map of the spinal cord laminae and their neurochemical content using the marker acetylcholinesterase and immunohistochemistry for substances such as calbindin $(\mathrm{Cb})$, calcitonin gene-related peptide (CGRP), calretinin (Cr), choline acetyltransferase (ChAT), enkephalin, neurofilament protein (SMI-32), and neuronal nuclear protein (NeuN) $[1,2]$.

The spinal dorsal horn is morphologically heterogeneous, but there is an intrinsic organization, so that zones of regularity or laminae can be identified. The ten laminae in the spinal cord gray matter based on cellular shape, size, and density were first described by Rexed in the cat $[3,4]$, followed by studies in the mouse $[2,5,6]$, rat $[2,7,8]$, marmoset, rhesus monkey, and human [2]. Six of these laminae (laminae 1-6) are in the dorsal horn. Neurons within a lamina often show distinct dendritic architecture, chemoarchitecture, and patterns of connections, as well as different functions. These neurons receive a variety of neurotransmitter inputs and release a variety of neurotransmitters that show different patterns of distribution that is often similar across different species.

Transverse sections of the spinal cord show the laminar distribution of the neurons, while sagittal sections are useful for the determination of the morphology of neurons and the distribution of their dendritic and axonal processes. Until recently, the only way to visualize the spinal cord in 3D was to obtain serial sections and make a reconstruction of them. However, tissue sectioning results in the breaking of axons 
and dendrites. Consequently, the broken axons and dendrites are difficult to be accurately realigned in $3 \mathrm{D}$ reconstruction.

CLARITY is a relatively novel technique developed by Karl Deisseroth and his colleagues that turns the brain transparent using the detergent sodium dodecyl sulfate by removing lipids that normally block the passage of light [9]. Unlike traditional histological methods where the tissue is sectioned for imaging, 3D imaging of the entire cleared tissue allows examination of labeled cells and molecules in the entire specimen, allowing marking and visualization of long-range projections and subcellular structures. The other advantage of CLARITY is the application of lipophilic dyes to trace neurons and their projections in the cleared tissue [1].

Thus far, the only studies using CLARITY in the spinal cord have examined the distribution of neuronal calciumbinding proteins 1 and 2 (NECABB1/2) [10] and serotonergic fibers [11]. In this study, we aimed to show the cytoand chemoarchitecture of the mouse spinal cord dorsal horn with many protein markers, i.e., calretinin, glycine, nitric oxide, CGRP, ChAT, GAD67, and serotonin 3D using CLARITY for the first time.

\section{Materials and Methods}

2.1. Animals. The animal work was approved by the Animal Care and Ethics Committee (ACEC) at The University of New South Wales (approval number 14/94A). Experiments were conducted on C57BL/6J $(n=12)$ male mice (http:// jaxmice.jax.org/strain/013636.html) and transgenic (ChAT:eGFP, $n=3$ ) male mice (https://www.jax.org/strain/ 007902) expressing enhanced green fluorescent protein in cholinergic neurons.

2.2. Tissue Clearing. Mice were anaesthetized with a lethal dose of pentobarbital solution $(0.1 \mathrm{ml}, 200 \mathrm{mg} / \mathrm{ml})$ and perfused with ice-cold hydrogel solution containing $4 \%$ acrylamide, $0.05 \%$ Bis, $0.025 \%$ VA-044 initiator, and 4\% PFA in PBS, following the protocol of Chung et al. [9]. After removal, the spinal cord was cut into $2-3 \mathrm{~mm}$ segments and kept in $5 \mathrm{ml}$ plastic tubes at $4^{\circ} \mathrm{C}$ overnight on a rotator. Subsequently, the tissue was degassed by replacing the air with nitrogen in a fume hood for $10-15 \mathrm{~min}$. The tissue was then transferred to a $37^{\circ} \mathrm{C}$ oven for incubation on a rotator. After the solution became a hydrogel, the tissue was removed from the gel and washed with the clearing solution $(0.2 \mathrm{M}$ boric acid, $4 \%$ sodium dodecyl sulfate, $\mathrm{pH}$ 8.5) for 7-10 d until the tissue became transparent.

2.3. Immunofluorescence Staining. The following procedure was modified from a protocol by Tomer et al. [12]. After washing the lumbar cord tissue with PBST $(0.1 \%$ Triton X$100)$ every $6 \mathrm{~h}$ for $24 \mathrm{~h}$, the tissue was incubated in primary antibody solutions $(1: 100)$ for $3 \mathrm{~d}$ on a rotator at $37^{\circ} \mathrm{C}$. For primary antibodies, we used rabbit anti-calretinin (Merck Millipore, AB5054), rabbit anti-CGRP (Merck Millipore, Cat\# AB15360), goat anti-ChAT (Merck Millipore, Cat\# AB144P), rabbit anti-serotonin (Sigma, Cat\# S5545), sheep anti-glycine transporter 2 neuronal (Merck Millipore, Cat\# AB1771), mouse anti-GAD67 (clone 1G10.2; Merck Milli- pore, Cat\# MAB5406), rabbit anti-nitric oxide synthase (anti-NOS, Merck Millipore, Cat\# AB5380), and rabbit anti-GABA (Sigma, Cat\# A2052). The tissue was subsequently washed with PBST every $6 \mathrm{~h}$ for $24 \mathrm{~h}$ and incubated in secondary antibodies, Alexa Fluor 594 conjugated goat anti-rabbit IgG (Life Technologies, Cat\# A-11012), Alexa Fluor 594 conjugated goat anti-mouse IgG (Life Technologies, Cat\# A-11032), Alexa Fluor 594 conjugated donkey anti-goat IgG (Life Technologies, Cat\# A-11058), or Alexa Fluor 594 conjugated donkey anti-sheep IgG (Life Technologies, Cat\# A-11016) for 3 days at $37^{\circ} \mathrm{C}$, at $1: 100$ dilution each, before they were washed again with PBST. After $24 \mathrm{~h}$, the tissue was put into $85 \%$ glycerol for homogenizing the refractive index before imaging.

Spinal cord tissues were imaged with a Leica TCS SP5 multiphoton microscope (Leica Microsystems $\mathrm{GmbH}$, Wetzlar, Germany) using 20x and 63x objectives. Leica TCS SP5 II has four lasers with the following lines for excitation: diode $50 \mathrm{~mW}$ with $405 \mathrm{~nm}$ emission line, "blue" argon multiline $65 \mathrm{~mW}$ with 458/476/488/514 nm emission lines, "yellow" diode $20 \mathrm{~mW}$ with $561 \mathrm{~nm}$ emission line, and "red" $10 \mathrm{~mW}$ with $633 \mathrm{~nm}$ emission line. Five channels, including a TLD brightfield detector, an internal detector channel (PMT), and three internal GaAsP detectors (HyD), were available to use. For 20x imaging, HC PL APO 20x/0.70 IMM CORR CS, $\mathrm{H}_{2} \mathrm{O} /$ glycerol/oil, objective numerical aperture was 0.70 , resolution $X Y: 279$, resolution $Z: 1284$, and free working distance: 260 . For 63x imaging, HCX PL APO $63 \mathrm{x} / 1.30 \mathrm{GLYC} \mathrm{CORR} \mathrm{CS} 21^{\circ} \mathrm{C}$, objective numerical aperture was 1.3 , resolution $X Y: 160$, resolution $Z: 335$, and free working distance: 280. IC Prisms: C; C1-P; C1; Cond. Prism DIC: K3, K6, 405; correction optic: L1, and UV correction optic: L1. IC Prisms: C; C1-P; C1, Cond. Prism DIC: K3, K6, 405 correction optic: L1, and UV correction optic: L1. The image was scanned at $400 \mathrm{~Hz}$ speed at zoom 1 and resolution of $1024 \times 1024$ pixels $(760 \mathrm{~nm} \times 760 \mathrm{~nm})$ with a step size of $3 \mu \mathrm{m}$ for the $20 \mathrm{x}$ objective and $1 \mu \mathrm{m}$ for the $63 \mathrm{x}$ objective. Emitted signals were collected into hybrid detectors (HD) with line accumulation of $4-8 x$. In the present study, the mouse lumbar cord was cut into small segments, but the imaging depth was limited by the working distance of the microscope, which is less than $300 \mu \mathrm{m}$. 3D reconstructions were made using OsiriX Imaging Software (Pixmeo Labs., Geneva, Switzerland) as described by Rosset et al. [13].

\section{Results}

3.1. General Findings. This distribution of multiple proteins, including calretinin, NOS, CGRP, ChAT, GAD67, GABA, serotonin, and glycine using CLARITY. Our observations of the cyto- and chemoarchitecture of the dorsal horn were similar to earlier reports that used conventional light and fluorescent microscopy techniques (Figures 1 and 2, Online resources 1-6), thus confirming that CLARITY can be used reliably to examine spinal cord tissue. These findings are summarized in Table 1. Briefly, ChAT, CGRP, calretinin, GABA, GAD67, GlyT2, and NOS immunoreactive neurons were observed across all laminae of the dorsal horn in the depth as long as the working distance of the microscope. 

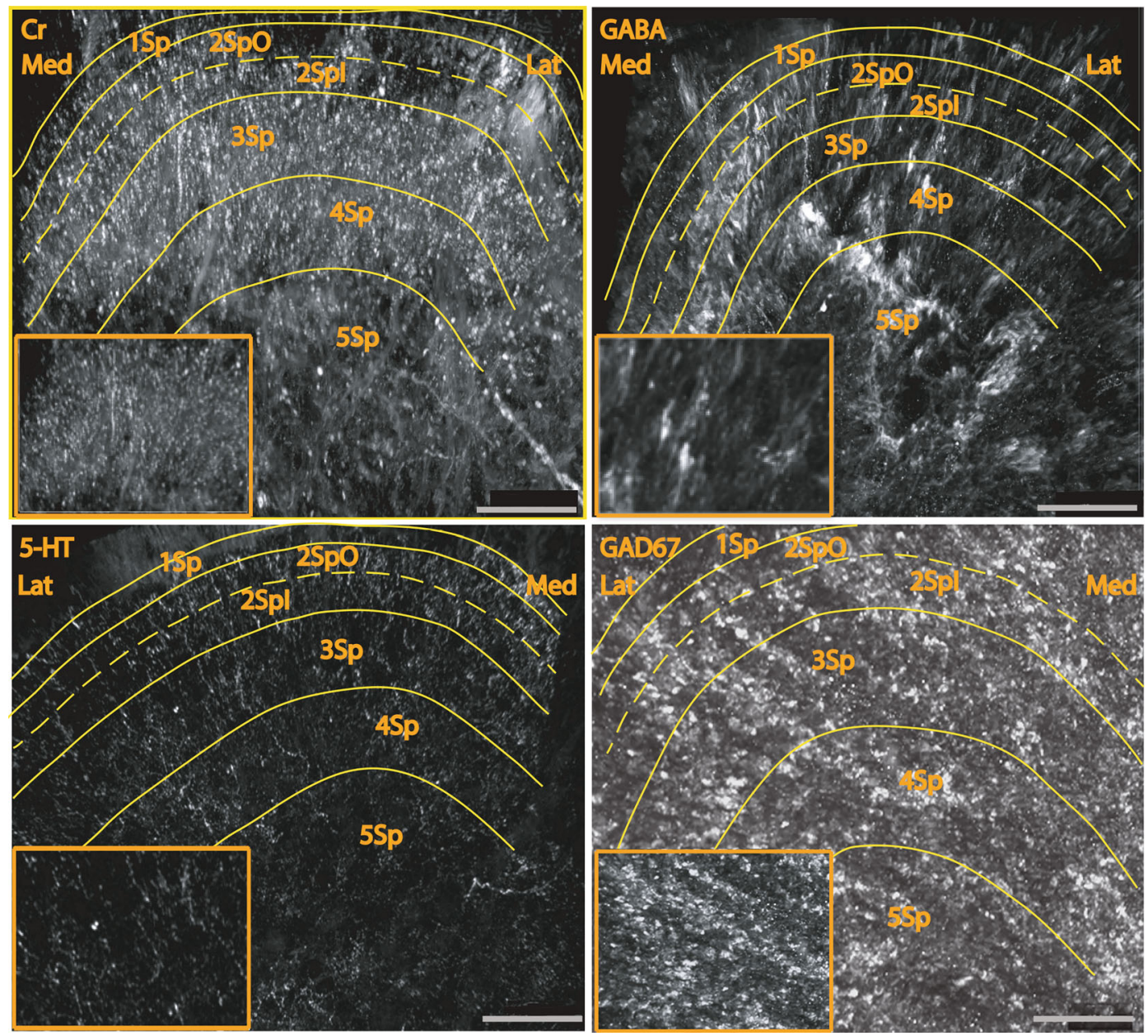

Figure 1: Cr (segment L1), GABA (segment L2), serotonin (segment L2), and GAD67 (segment L3) immunoreactive neurons and fibers in the mouse spinal dorsal horn. Scale bar is $100 \mu \mathrm{m}$. Med: medial; Lat: lateral.

Serotonergic perikarya were not observed. The borders of each lamina were defined by cell size and density, using an atlas of the mouse spinal cord [1] constructed on cyto- and chemoarchitecture of Rexed's laminae.

\subsection{Novel Findings Different from $2 D$ Imaging. Our findings} are mostly consistent with those of prior studies (as outlined in Table 1; also see Discussion for details). However, there were some novel observations for CGRP, ChAT, GABA, GAD67, and serotonin that we have identified using CLARITY technique.

Firstly, we observed numerous CGRP immunoreactive perikarya in all laminae along with CGRP fibers and terminals, which were densest in laminae 1 and 4-6 (Figure 2, Online resources 1). Secondly, many small ChAT perikarya were also found in all laminae, and sparse ChAT immunoreactivity was found for fibers and terminals throughout the dorsal horn after ChAT antibody labeling (Figure 2, Online resources 4). Similar findings were observed in the ChATGFP mouse lumbar cord. Finally, GABA and GAD67 immunoreactivities were found throughout the dorsal horn in small perikarya. GABA and GAD67 immunoreactive fibers were found more densely in laminae 1-4 and attenuated in laminae 5-6. For GABA, we also found antenna-like neurons (with dendrites extending dorsally) in lamina 3 (Figure 2, Online resources 2 and 3 ), an observation not previously reported. Serotonergic fibers were observed in all laminae of the dorsal horn (Figure 1, Online resources 5).

\section{Discussion}

Over the last several decades, many studies have shed light on the cyto- and chemoarchitecture of the spinal dorsal horn using a variety of techniques, especially Golgi staining, intracellular injection methods, and histochemical/immunohistochemical stains. However, these require tissue sectioning and imaging of individual sections to make reconstructions in order to see cell morphology and cytoarchitecture in detail-an approach limited by loss of tissue during sectioning, mechanical distortions, and difficulties in the alignment of the images. In the present study, the cyto- and chemoarchitecture of the mouse spinal cord dorsal horn have been revealed for the first time in $3 \mathrm{D}$, using a variety of immunohistochemical protein markers with unprecedented detail. CLARITY can provide more accurate representations of subtle anatomical characteristics in the spinal cord and provide $3 \mathrm{D}$ videos for observation. A recent study by Ertürk et al. [14] used a tetrahydrofuran-based clearing technique to 

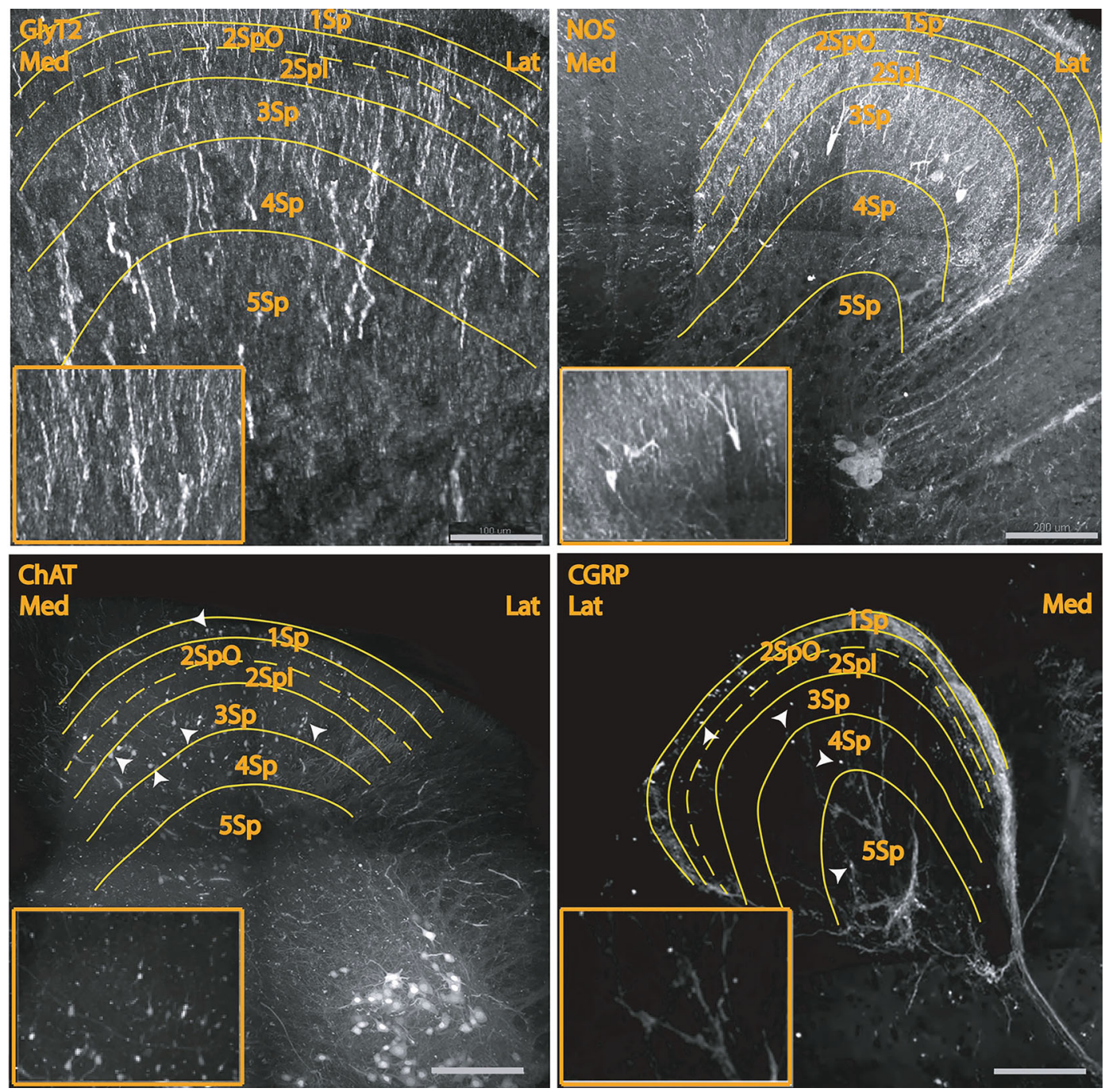

FIGURE 2: GlyT2 (segment L2), NOS (segment L2), ChAT (segment L5), and CGRP (segment L2) immunoreactive perikarya (arrowheads) and fibers in the superficial laminae of the mouse spinal dorsal horn. Scale bar: $100 \mu \mathrm{m}$ for GlyT2 and $200 \mu \mathrm{m}$ for NOS, CHAT, and CGRP. Med: medial; Lat: lateral.

TABle 1: Distribution of CGRP, ChAT, Cr, GABA, GAD67, GlyT2, NOS, and serotonin immunoreactive perikarya in the mouse spinal cord using CLARITY.

\begin{tabular}{lcccccc}
\hline Lamina & 1 & 2 & 3 & 4 & 5 & 6 \\
\hline CGRP & ++ & + & + & + & + & + \\
ChAT & + & ++ & + & + & + & + \\
Cr & ++ & ++ & ++ & + & + & + \\
GABA & ++ & ++ & ++ & ++ & ++ & ++ \\
GAD67 & ++ & ++ & ++ & ++ & ++ & ++ \\
GlyT2 & ++ & ++ & ++ & ++ & ++ & ++ \\
NOS & ++ & ++ & ++ & + & + & + \\
Serotonin & - & - & - & - & - & - \\
\hline
\end{tabular}

visualize microglia, astrocytes, and neurons in eGFP mice and determined the cell number and axonal regeneration following spinal cord injury. However, they did not apply any immunohistochemical markers or produce data on the cytoand chemoarchitecture of the spinal cord.

In the present study, we observed CGRP, ChAT, GAD67, GABA, serotonin, and glycine immunoreactivities in the mouse spinal cord for the first time using the CLARITY technique. Our observations with CLARITY support earlier reports on the overall density and immunohistochemical localization of spinal cord neurons in the mouse for calretinin [15], GABA $[16,17]$, and NOS $[18,19]$ and in the rat for calretinin [20, 21], GABA [22-24], glycine [25-28], and NOS $[19,29,30]$. Replicating prior studies that used standard $2 \mathrm{D}$ imaging, and extending these with observations in $3 \mathrm{D}$ using CLARITY, is important in validating the results of this technique. This suggests that future studies can move forward with CLARITY. Finally, we observed new details for CGRP, ChAT, GABA, GAD67, and serotonin as discussed below.

4.1. CGRP. With CLARITY, we were able to identify numerous CGRP immunoreactive perikarya in all laminae of the 
mouse dorsal horn. CGRP fibers and terminals were also found in all laminae, denser in laminae 1 and 4-6 (Figure 2, Online resources 1 ). In studies with $2 \mathrm{D}$ immunohistochemical staining, CGRP fibers and terminals were shown mainly in laminae 1-2 and to a lesser extent in laminae 3-4 and 6 of the mouse spinal cord [31-33]. CGRP immunoreactive perikarya were first reported by Conrath et al. [34], encountered only rarely in lamina 1 , more in lamina 2 , and most frequently in lamina 3 of the rat spinal cord. Tie-Jun et al. [32] observed much fewer CGRP perikarya in the mouse, only in lamina 3. Our findings suggest that, although the major origin of CGRP in the spinal cord is from afferent fibers derived from dorsal root ganglion neurons, CGRP immunoreactive perikarya throughout the dorsal horn laminae also contribute to the sensory functions of the spinal cord including nociception. Lately, it has been suggested that CGRP facilitates nociceptive transmission and maintains central sensitization in both the primary afferent sensory neurons and the secondorder pain transmission neurons within the central nervous system $[35,36]$. A denser distribution of CGRP fibers in lamina 4 indicates that these laminae receive more terminals from primary sensory neurons. Given that lamina 4 is recognized to receive proprioceptive input [1], this suggests a possible role for CGRP neurons of the dorsal horn in nociceptive and nonnociceptive sensory information including proprioception. CGRP immunoreactive perikarya throughout the dorsal horn laminae presumably play a role in the sensory functions of the spinal cord including nociception.

4.2. ChAT. With CLARITY, we observed sparse ChAT immunoreactivity in fibers and many small perikarya in all laminae of the dorsal horn of the ChAT:eGFP transgenic mice (Figure 2, Online resources ChAT). Cholinergic neurons in the midbrain do not make direct connections with the spinal cord, and this is consistent with the absence of ChAT and VAChT immunolabeling in the spinal white matter [37]. This suggests that cholinergic fibers in the dorsal horn are either derived from intraspinal cholinergic neurons or from primary sensory neurons. Intraspinal cholinergic neurons and primary sensory neurons are the primary sources of cholinergic fibers in the spinal cord. Supporting this, cholinergic neurons in the midbrain do not have any synaptic connections with the rat spinal cord neurons [37]. A dense plexus of cholinergic fibers is found in laminae 2 and 3 of the rat spinal cord $[38,39]$, but no cytoplasmic immunoreactivity has been shown for ChAT or vesicular acetylcholine transporter (VAChT) in the rat, mouse, and primate spinal cords [40-42]. However, some studies reported a large number of ChAT immunoreactive neurons in laminae 3-4 of the rat $[38,43]$. Recently, a study on ChAT-eGFP mice also showed a low number of perikarya $(2.8 \pm 0.3$ perikarya per $50 \mu \mathrm{m}$ thick transverse section in laminae 3-4) [44]. As well as laminae 3-4, we have observed a dense cholinergic plexus in lamina $2 \mathrm{i}$ and less dense in laminae 1 and 2o. The presence of ChAT perikarya beyond laminae 3-4 indicates that ChAT has a wider range of functions in the spinal cord than previously thought. For example, ChAT is likely to modulate both nonnociceptive and nociceptive sensory information including inhibition of nocicep- tive transmission given that these are recognized functions of laminae 1 and 2 [45].

4.3. GAD67 and GABA. With CLARITY, we observed GABA and GAD67 immunoreactivities in all laminae of the dorsal horn both in fibers and small perikarya. GABA and GAD67 immunoreactive fibers were dense in laminae 1-4 and moderately dense in laminae 5-6. For GABA, we also identified antenna-like neurons that have dorsally oriented dendrites in lamina 3 (Figure 1, Online resources GAD, GABA). GABA-containing neurons within the spinal dorsal horn were initially identified in laminae 1-3 of the rat using glutamate decarboxylase (GAD) immunohistochemical staining [46-49]. The majority of these cells had ventrally oriented dendrites. Also, GABA immunoreactivity was found in small fusiform, round, or multipolar-shaped perikarya mainly in laminae 1-3 and fewer in laminae 4-6 [22, 50-52] of the rat spinal cord. The present study confirms these observations and additionally demonstrates GABA neurons in laminae 5-6 using CLARITY.

4.4. Serotonin. In the mouse, all serotonergic cell groups of the caudal hindbrain have projections to the spinal cord [41] and play a modulatory role in the central regulation of many autonomic functions. Previously, serotonergic fibers were observed in all laminae, being most abundant in laminae $2 \mathrm{i}$ and $2 \mathrm{o}[1,6,52]$. With CLARITY, we observed numerous serotonergic fibers in all laminae of the dorsal horn. Similarly, in a recent study, we have observed serotonergic fibers in all laminae of the dorsal horn, excluding the lateral part of laminae 2 and 4 [11].

\section{Conclusions}

With the CLARITY clearing technique, we observed the cyto- and chemoarchitecture of the mouse spinal cord in 3D for the first time for calretinin, CGRP, ChAT, serotonin, glycine, GAD67, NOS, and GABA. We confirmed prior observations of the locations of these proteins in spinal cord laminae and reported novel observations of visualization of the proteins of interest in $3 \mathrm{D}$, concluding that CLARITY is a suitable technique for visualization of the spinal cord in unprecedented detail.

\section{Data Availability}

All results produced from the present study are available upon reasonable request to the corresponding author.

\section{Disclosure}

The funders had no role in the design of the study; in the collection, analyses, or interpretation of data; in the writing of the manuscript; or in the decision to publish the results.

\section{Conflicts of Interest}

The authors declare no conflict of interests. 


\section{Authors' Contributions}

Conceptualization, investigation, and writing (original draft preparation) were handled by GS and HL; methodology and formal analysis were made by GS, HL, and TF; software was taken care of by TF; validation was done by GS, HL, and GP; resources and supervision were managed by GP; data curation was tended by HL and TF; writing (review and editing) was conducted by TF and GP; visualization was addressed by GS; project administration was directed by HL; and funding acquisition was headed by HL and GP. All authors have read and agreed to the published version of the manuscript.

\section{Acknowledgments}

We thank Dr. Emma Schofield for her technical support. This research was funded by the National Health and Medical Research Council (APP1140295 and APP1188744, awarded to George Paxinos), Scientific and Technological Research Council of Turkey (awarded to Gulgun Sengul, SBAG-114S405), Rebecca L. Cooper Medical Research Foundation (no grant number available, awarded to Huazheng Liang), and Centre of Excellence for Integrative Brain Function, Australian Research Council (CE140100007, awarded to George Paxinos), and the APC was funded by the Rebecca L. Cooper Medical Research Foundation.

\section{Supplementary Materials}

Supplementary 1. Online resources 1: distribution of CGRPpositive fibers in the dorsal horn of the mouse L2 lumbar cord. The top is dorsal, and the right is medial.

Supplementary 2. Online resources 2: distribution of GAD67positive fibers in the dorsal horn of the mouse L3 lumbar cord. The top is dorsal, and the right is medial.

Supplementary 3. Online resources 3: distribution of GABApositive fibers in the dorsal horn of the mouse L2 lumbar cord. The top is dorsal, and the right is lateral.

Supplementary 4. Online resources 4: distribution of ChATpositive fibers in the dorsal horn of the mouse L5 lumbar cord. The left is dorsal, and the top is lateral.

Supplementary 5. Online resources 5: distribution of serotonin-positive fibers in the dorsal horn of the mouse L2 lumbar cord. The left is dorsal, and the top is lateral.

Supplementary 6. Online resources 6: distribution of glycinepositive fibers in the dorsal horn of the mouse L2 lumbar cord. The top is dorsal, and the right is lateral.

\section{References}

[1] C. Heise and G. Kayalioglu, "Cytoarchitecture of the spinal cord," in The Spinal Cord. A Christopher and Dana Reeve Foundation Text and Atlas, C. Watson, G. Paxinos, and G. Kayalioglu, Eds., pp. 64-80, Academic Press Elsevier, San Diego, USA, 1st edition, 2009.
[2] G. Sengul, C. Watson, I. Tanaka, and G. Paxinos, Atlas of the Spinal Cord: Mouse, Rat, Rhesus, and Human, Academic Press Elsevier, San Diego, USA, 2013.

[3] B. Rexed, "The cytoarchitectonic organization of the spinal cord in the cat," The Journal of Comparative Neurology, vol. 96, no. 3, pp. 414-495, 1952.

[4] B. Rexed, "A cytoarchitectonic atlas of the spinal cord in the cat," The Journal of Comparative Neurology, vol. 100, no. 2, pp. 297-379, 1954

[5] R. L. Sidman, J. B. Angevine Jr., and E. T. Pierce, Atlas of the Mouse Brain and Spinal Cord, Harvard University Press, Cambridge, MA, USA, 1971.

[6] C. Heise and G. Kayalioglu, "Chapter 13 - Spinal cord transmitter substances," in The Spinal Cord. A Christopher and Dana Reeve Foundation Text and Atlas, C. Watson, G. Paxinos, and G. Kayalioglu, Eds., pp. 191-198, Academic Press Elsevier, San Diego, USA, 1st edition, 2009.

[7] C. Molander, Q. Xu, and G. Grant, "The cytoarchitectonic organization of the spinal cord in the rat. I. The lower thoracic and lumbosacral cord," The Journal of Comparative Neurology, vol. 230, no. 1, pp. 133-141, 1984.

[8] C. Molander, Q. Xu, C. Rivero-Melian, and G. Grant, "Cytoarchitectonic organization of the spinal cord in the rat: II. The cervical and upper thoracic cord," The Journal of Comparative Neurology, vol. 289, no. 3, pp. 375-385, 1989.

[9] K. Chung, J. Wallace, S. Y. Kim et al., "Structural and molecular interrogation of intact biological systems," Nature, vol. 497, no. 7449, pp. 332-337, 2013.

[10] M. D. Zhang, G. Tortoriello, B. Hsueh et al., "Neuronal calcium-binding proteins $1 / 2$ localize to dorsal root ganglia and excitatory spinal neurons and are regulated by nerve injury," Proceedings of the National Academy of Sciences of the United States of America, vol. 111, no. 12, pp. E1149E1158, 2014.

[11] H. Liang, E. Schofield, and G. Paxinos, "Imaging serotonergic fibers in the mouse spinal cord using the CLARITY/CUBIC technique," Journal of Visualized Experiments, vol. 108, 2016.

[12] R. Tomer, L. Ye, B. Hsueh, and K. Deisseroth, "Advanced CLARITY for rapid and high-resolution imaging of intact tissues," Nature Protocols, vol. 9, no. 7, pp. 1682-1697, 2014.

[13] A. Rosset, L. Spadola, and O. Ratib, "OsiriX: an open-source software for navigating in multidimensional DICOM images," Journal of Digital Imaging, vol. 17, no. 3, pp. 205-216, 2004.

[14] A. Ertürk, C. P. Mauch, F. Hellal et al., "Three-dimensional imaging of the unsectioned adult spinal cord to assess axon regeneration and glial responses after injury," Nature Medicine, vol. 18, no. 1, pp. 166-171, 2011.

[15] X. Fan, H. J. Kim, M. Warner, and J. A. Gustafsson, "Estrogen receptor beta is essential for sprouting of nociceptive primary afferents and for morphogenesis and maintenance of the dorsal horn interneurons," Proceedings of the National Academy of Sciences of the United States of America, vol. 104, no. 34, pp. 13696-13701, 2007.

[16] A. J. Kaduri, R. Magoul, L. Lescaudron, G. Campistron, and A. Calas, "Immunocytochemical approach of GABAergic innervation of the mouse spinal cord using antibodies to GABA," Journal für Hirnforschung, vol. 28, no. 3, pp. 349355, 1987.

[17] B. Heinke, R. Ruscheweyh, L. Forsthuber, G. Wunderbaldinger, and J. Sandkühler, "Physiological, neurochemical and morphological properties of a subgroup of GABAergic spinal lamina II 
neurones identified by expression of green fluorescent protein in mice," The Journal of Physiology, vol. 560, no. 1, pp. 249266, 2004.

[18] G. Brüning, "Localization of NADPH diaphorase, a histochemical marker for nitric oxide synthase, in the mouse spinal cord," Acta Histochemica, vol. 93, no. 2, pp. 397-401, 1992.

[19] N. J. Dun, S. L. Dun, S. Y. Wu, U. Förstermann, H. H. Schmidt, and L. F. Tseng, "Nitric oxide synthase immunoreactivity in the rat, mouse, cat and squirrel monkey spinal cord," Neuroscience, vol. 54, no. 4, pp. 845-857, 1993.

[20] K. Ren and M. A. Ruda, "A comparative study of the calciumbinding proteins calbindin- D28K, calretinin, calmodulin and parvalbumin in the rat spinal cord," Brain Research Brain Research Reviews, vol. 19, no. 2, pp. 163-179, 1994.

[21] R. Anelli and C. J. Heckman, "The calcium binding proteins calbindin, parvalbumin, and calretinin have specific patterns of expression in the gray matter of cat spinal cord," Journal of Neurocytology, vol. 34, no. 6, pp. 369-385, 2005.

[22] A. J. Todd and J. McKenzie, "GABA-immunoreactive neurons in the dorsal horn of the rat spinal cord," Neuroscience, vol. 31, no. 3, pp. 799-806, 1989.

[23] R. C. Spike and A. J. Todd, "Ultrastructural and immunocytochemical study of lamina II islet cells in rat spinal dorsal horn," The Journal of Comparative Neurology, vol. 323, no. 3, pp. 359-369, 1992.

[24] A. J. Todd and R. C. Spike, "The localization of classical transmitters and neuropeptides within neurons in laminae I-III of the mammalian spinal dorsal horn," Progress in Neurobiology, vol. 41, no. 5, pp. 609-645, 1993.

[25] G. Campistron, R. M. Buijs, and M. Geffard, "Glycine neurons in the brain and spinal cord. Antibody production and immunocytochemical localization," Brain Research, vol. 376, no. 2, pp. 400-405, 1986.

[26] A. N. Van den Pol and T. Gorcs, "Glycine and glycine receptor immunoreactivity in brain and spinal cord," The Journal of Neuroscience, vol. 8, no. 2, pp. 472-492, 1988.

[27] A. J. Todd, G. Russell, and R. C. Spike, "Immunocytochemical evidence that GABA and neurotensin exist in different neurons in laminae II and III of rat spinal dorsal horn," Neuroscience, vol. 47, no. 3, pp. 685-691, 1992.

[28] M. Hossaini, P. J. French, and J. C. Holstege, "Distribution of glycinergic neuronal somata in the rat spinal cord," Brain Research, vol. 1142, pp. 61-69, 2007.

[29] S. Saito, G. J. Kidd, B. D. Trapp et al., "Rat spinal cord neurons contain nitric oxide synthase," Neuroscience, vol. 59, no. 2, pp. 447-456, 1994.

[30] V. A. Maiskii, G. Kayaliolu, F. Govsa, B. Erdem, and N. I. Hariri, "Nitric oxide contributes to the spinal nociceptive processing," Neurophysiology, vol. 30, no. 6, pp. 370-374, 1998.

[31] S. J. Gibson, J. M. Polak, P. Anand et al., "The distribution and origin of VIP in the spinal cord of six mammalian species," Peptides, vol. 5, no. 2, pp. 201-207, 1984.

[32] S. S. Tie-Jun, Z. Xu, and T. Hökfelt, "The expression of calcitonin gene-related peptide in dorsal horn neurons of the mouse lumbar spinal cord," Neuroreport, vol. 12, no. 4, pp. 739-743, 2001.

[33] P. Brumovsky, D. Stanic, S. Shuster, H. Herzog, M. Villar, and T. Hökfelt, "Neuropeptide Y2 receptor protein is present in peptidergic and nonpeptidergic primary sensory neurons of the mouse," The Journal of Comparative Neurology, vol. 489, no. 3, pp. 328-348, 2005.
[34] M. Conrath, H. Taquet, M. Pohl, and A. Carayon, "Immunocytochemical evidence for calcitonin gene-related peptidelike neurons in the dorsal horn and lateral spinal nucleus of the rat cervical spinal cord," Journal of Chemical Neuroanatomy, vol. 2, no. 6, pp. 335-347, 1989.

[35] S. Iyengar, M. H. Ossipov, and K. W. Johnson, "The role of calcitonin gene-related peptide in peripheral and central pain mechanisms including migraine," Pain, vol. 158, pp. 543$559,2017$.

[36] G. O. Dussor, G. Helesic, K. M. Hargreaves, and C. M. Flores, "Cholinergic modulation of nociceptive responses in vivo and neuropeptide release in vitro at the level of the primary sensory neuron," Pain, vol. 107, pp. 22-32, 2004.

[37] R. P. Barber, P. E. Phelps, C. R. Houser, G. D. Crawford, P. M. Salvaterra, and J. E. Vaughn, "The morphology and distribution of neurons containing choline acetyltransferase in the adult rat spinal cord: an immunocytochemical study," The Journal of Comparative Neurology, vol. 229, no. 3, pp. 329346, 1984.

[38] M. J. Olave, N. Puri, R. Kerr, and D. J. Maxwell, "Myelinated and unmyelinated primary afferent axons form contacts with cholinergic interneurons in the spinal dorsal horn," Experimental Brain Research, vol. 145, no. 4, pp. 448-456, 2002.

[39] M. L. Gilmor, N. R. Nash, A. Roghani et al., "Expression of the putative vesicular acetylcholine transporter in rat brain and localization in cholinergic synaptic vesicles," The Journal of Neuroscience, vol. 16, no. 7, pp. 2179-2190, 1996.

[40] L. Kus, E. Borys, Y. Ping Chu et al., "Distribution of high affinity choline transporter immunoreactivity in the primate central nervous system," The Journal of Comparative Neurology, vol. 463, no. 3, pp. 341-357, 2003.

[41] V. G. J. M. Vander Horst and B. Ulfhake, "The organization of the brainstem and spinal cord of the mouse: relationships between monoaminergic, cholinergic, and spinal projection systems," Journal of Chemical Neuroanatomy, vol. 31, no. 1, pp. 2-36, 2006.

[42] C. R. Houser, G. D. Crawford, R. P. Barber, P. M. Salvaterra, and J. E. Vaughn, "Organization and morphological characteristics of cholinergic neurons: an immunocytochemical study with a monoclonal antibody to choline acetyltransferase," Brain Research, vol. 266, no. 1, pp. 97-119, 1983.

[43] B. Mesnage, S. Gaillard, A. G. Godin et al., "Morphological and functional characterization of cholinergic interneurons in the dorsal horn of the mouse spinal cord," The Journal of Comparative Neurology, vol. 519, no. 16, pp. 3139-3158, 2011.

[44] A. Ribeiro-da-Silva and A. C. Cuello, "Choline acetyltransferase-immunoreactive profiles are presynaptic to primary sensory fibers in the rat superficial dorsal horn," The Journal of Comparative Neurology, vol. 295, no. 3, pp. 370384, 1990.

[45] B. J. McLaughlin, R. Barber, K. Saito, E. Roberts, and J. Y. Wu, "Immunocytochemical localization of glutamate decarboxylase in rat spinal cord," The Journal of Comparative Neurology, vol. 164, no. 3, pp. 305-321, 1975.

[46] R. P. Barber, J. E. Vaughn, K. Saito, B. J. McLaughlin, and E. Roberts, "GABAergic terminals are presynaptic to primary afferent terminals in the substantia gelatinosa of the rat spinal cord," Brain Research, vol. 141, no. 1, pp. 35-55, 1978.

[47] R. P. Barber, J. E. Vaughn, and E. Roberts, “The cytoarchitecture of gabaergic neurons in rat spinal cord," Brain Research, vol. 238, no. 2, pp. 305-328, 1982. 
[48] S. P. Hunt, J. S. Kelly, P. C. Emson, J. R. Kimmel, R. J. Miller, and J. Y. Wu, "An immunohistochemical study of neuronal populations containing neuropeptides or gammaaminobutyrate within the superficial layers of the rat dorsal horn," Neuroscience, vol. 6, no. 10, pp. 1883-1898, 1981.

[49] R. Magoul, B. Onteniente, M. Geffard, and A. Calas, "Anatomical distribution and ultrastructural organization of the GABAergic system in the rat spinal cord. An immunocytochemical study using anti-GABA antibodies," Neuroscience, vol. 20, no. 3, pp. 1001-1009, 1987.

[50] A. J. Todd and A. C. Sullivan, "Light microscope study of the coexistence of GABA-like and glycine-like immunoreactivities in the spinal cord of the rat," The Journal of Comparative Neurology, vol. 296, no. 3, pp. 496-505, 1990.

[51] D. Lima and A. Coimbra, "Morphological types of spinomesencephalic neurons in the marginal zone (lamina I) of the rat spinal cord, as shown after retrograde labelling with cholera toxin subunit B," The Journal of Comparative Neurology, vol. 279, no. 2, pp. 327-339, 1989.

[52] C. Sur, H. Betz, and P. Schloss, "Localization of the serotonin transporter in rat spinal cord," The European Journal of Neuroscience, vol. 8, no. 12, pp. 2753-2757, 1996. 